\title{
In-season and out-of-season variation of rotavirus genotype distribution and age of infection across 12 European countries before the introduction of routine vaccination, $2007 / 08$ to $2012 / 13$
}

D Hungerford ${ }^{12}$, R Vivancos ${ }^{234}$, EuroRotaNet network members ${ }^{5}$, JM Read ${ }^{6}$, VE Pitzer ${ }^{7}, \mathrm{~N}_{\text {Cunliffe }}{ }^{18}$, N French ${ }^{19}$, M IturrizaGómara ${ }^{13}$

1. Department of Clinical Infection, Microbiology and Immunology, Institute of Infection and Global Health, University of Liverpool, Liverpool, United Kingdom

2. Public Health England, Field Epidemiology Services, Liverpool, United Kingdom

3. NIHR Health Protection Research Unit in Gastrointestinal Infections, University of Liverpool, United Kingdom

4. NIHR Health Protection Research Unit in Emerging and Zoonotic Infections, University of Liverpool, United Kingdom

5. Members of the network are listed at the end of the article

6. Department of Epidemiology and Population Health, Institute of Infection and Global Health, The Farr Institute@HeRC, University of Liverpool, Liverpool, United Kingdom

7. Department of Epidemiology of Microbial Diseases, Yale School of Public Health, New Haven, CT, United States

8. Alder Hey Children's NHS Foundation Trust, Liverpool, United Kingdom

9. Royal Liverpool and Broadgreen University Hospitals NHS Trust, Liverpool, United Kingdom

Correspondence: Daniel Hungerford (d.hungerford@liverpool.ac.uk)

Citation style for this article:

Hungerford D, Vivancos R, EuroRotaNet network members, Read J, Pitzer V, Cunliffe N, French N, Iturriza-Gómara M. In-season and out-of-season variation of rotavirus genotype distribution and age of infection across 12 European countries before the introduction of routine vaccination, $2007 / 08$ to $2012 / 13$. Euro Surveill. 2016;21(2):pii=30106. DOI: http://dx.doi.org/10.2807/1560-7917.ES.2016.21.2.30106

Article submitted on 15 April 2015 / accepted on 14 September 2015 / published on 14 January 2016

The EuroRotaNet surveillance network has conducted rotavirus genotype surveillance since 2007 in 16 European countries. Using epidemiological and microbiological data from 39,786 genotyped rotavirus-positive specimens collected between September 2007 and August 2013, we assessed genotype distribution and age distribution of rotavirus gastroenteritis (RVGE) cases in and out of peak season in 12 countries which were yet to implement routine rotavirus vaccination. In multinomial multivariate logistic regression, adjusting for year, country and age, the odds of infection caused by genotype-constellation 2 DS-1-like stains (adjusted multinomial odds ratio $(\mathrm{aM}-\mathrm{OR})=1.25 ; 95 \%$ confidence interval $(\mathrm{Cl})$ : 1.13-1.37; $p<0.001)$, mixed or untypable genotypes (aM-OR $=1.55 ; 95 \% \mathrm{Cl}: 1.40-1.72 ; \mathrm{p}<0.001)$ and less common genotypes $(\mathrm{aM}-\mathrm{OR}=\mathbf{2 . 1 1}$; 95\% Cl:1.78-2.51; $\mathrm{p}<0.001)$ increased out of season relative to $\mathrm{G}_{1} \mathrm{P}[8]$. Age varied significantly between seasons; the proportion of RVGE cases younger than 12 months in the United Kingdom increased from 34\% in season to $39 \%$ out of season (aM-OR =1.66;95\% Cl: 1.20-2.30), and the proportion five years and older increased from $9 \%$ in season to $17 \%$ out of season $(\mathrm{aM}-\mathrm{OR}=2.53 ; 95 \% \mathrm{Cl}$ : 1.67-3.82). This study provides further understanding of the rotavirus ecology before vaccine introduction, which will help interpret epidemiological changes in countries introducing or expanding rotavirus vaccination programmes.

\section{Introduction}

Rotavirus is the most common cause of acute gastroenteritis in children under five years of age, causing an estimated 450,000 deaths per year worldwide, with over $90 \%$ of deaths occurring in developing countries [1]. In high-income countries, rotavirus infections result in few deaths but still constitute a substantial healthcare burden and can cause severe morbidity $[2,3]$. There are eight groups of rotaviruses defined by the middle capsid antigen [4]; the majority of rotavirus gastroenteritis (RVGE) in humans is caused by group $A$ rotaviruses.

Group A rotavirus genotypes are typically further classified into $G$ and $P$ types, based on sequence diversity of the genes encoding the outer viral proteins VP7 (glycoprotein) and VP4 (protease-sensitive protein), respectively [5]. Furthermore, whole genome sequencing has allowed rotavirus strains to be classified into genotype constellations based on a common genomic backbone in which the genotypes of nine of the 11 genes are conserved, while $G$ and $P$ types may vary. Human rotaviruses typically belong to the Wa-like or the DS-1-like genotype constellations [6].

Two oral vaccines, the two-dose monovalent vaccine (Rotarix, GlaxoSmithKline Biologicals, Belgium) and the three-dose pentavalent vaccine (RotaTeq, Merck, United States), have been introduced into a number 
Number of rotavirus specimens typed per week by country and surveillance year, 12 European Union countries, September 2007-August $2013(\mathrm{n}=39,786)$

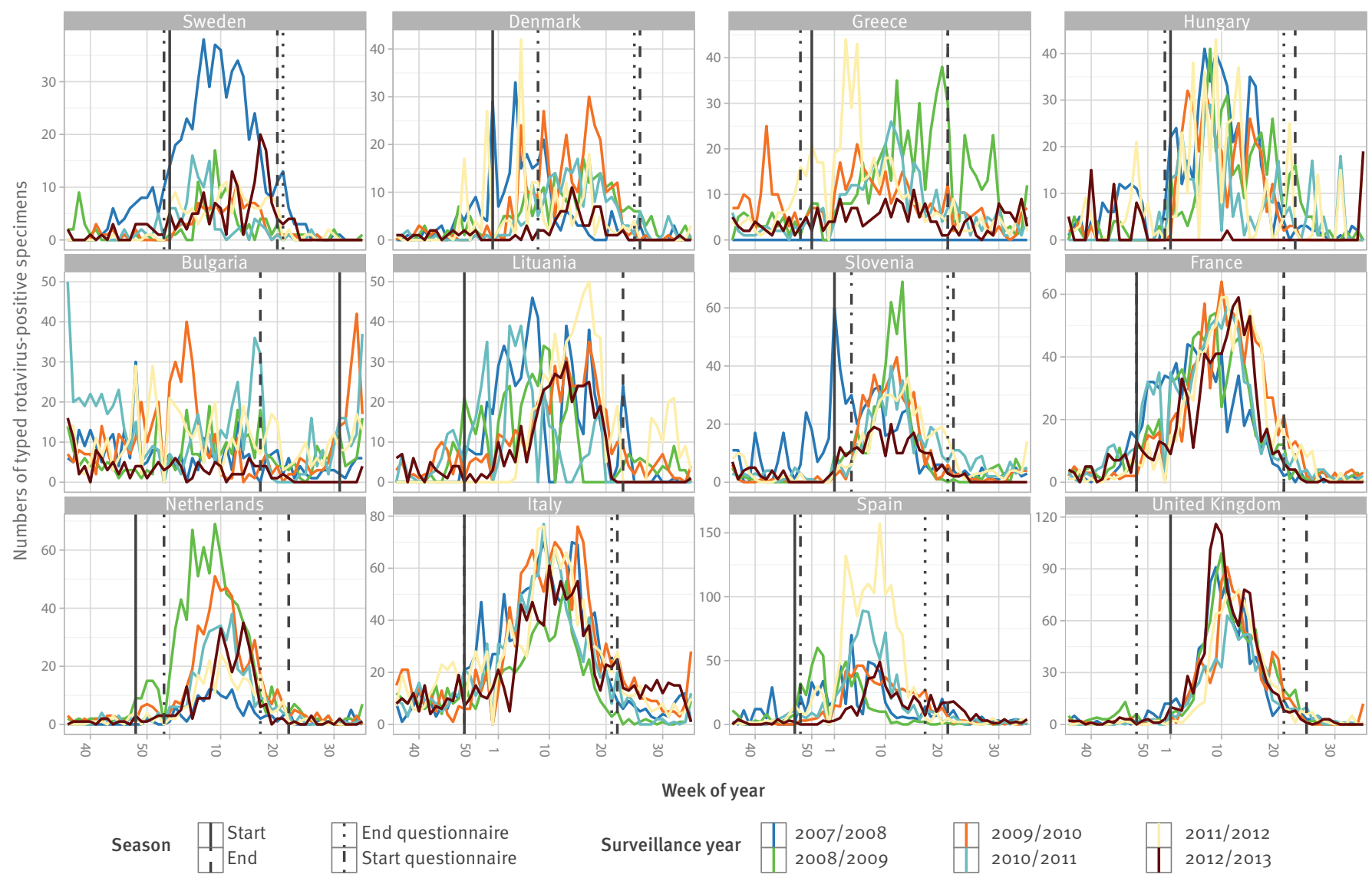

The season legend shows lines for the start and the end of the rotavirus season as defined by the median threshold method and reported through the questionnaire.

of countries worldwide since their licensure in 2006. Eight European Union countries have included rotavirus vaccines in their routine childhood immunisation schedules and several other countries make the vaccine available through state or private sector healthcare [7].

Monitoring the emergence of novel rotavirus genotypes and the potential for genotype replacement and genetic drift is an essential activity of surveillance. This has become more important since the introduction of rotavirus vaccination, as there was some early evidence in countries such as Australia, Brazil and Belgium that vaccination may have contributed to changes in the predominant genotypes, although these changes may also have been the result of natural variation $[8,9]$. The EuroRotaNet surveillance network, established in 2007 and including 16 countries, has been monitoring rotavirus genotype diversity and year-to-year genotype fluctuations across Europe for eight years $[10,11]$. Critically, the availability of substantial genotyping and epidemiological data for EuroRotaNet countries provides a baseline for genotype diversity and the epidemiology of RVGE cases before vaccine introduction. Therefore, while year-to-year differences in genotypes in Europe have been described previously $[11,12]$, this paper reports in-peak season and out-of-season variation of rotavirus genotypes and age of infection for 12 European countries before the introduction of routine vaccination.

\section{Methods}

\section{EuroRotaNet}

The EuroRotaNet surveillance network was initiated in 2007 and includes 16 countries: Austria, Belgium, Bulgaria, Denmark, Finland, France, Germany, Greece, Hungary, Italy, Lithuania, the Netherlands, Slovenia, Spain, Sweden and the United Kingdom (UK). Data from typed rotavirus-positive faecal specimens is linked to case epidemiological information by participating laboratories and uploaded to a secure webaccessible EuroRotaNet database. The data contained in the EuroRotaNet dataset has been described previously $[10,11]$. 


\section{FIGURE 2}

Number of rotavirus specimens collected in season and out of season, by country, 12 European Union countries, September 2007-August $2013(\mathrm{n}=39,786)$

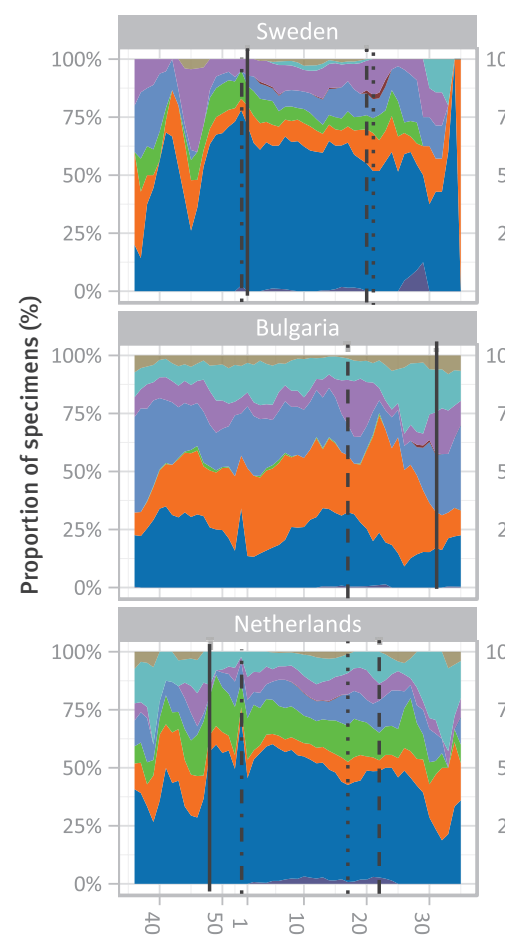

Genotypes

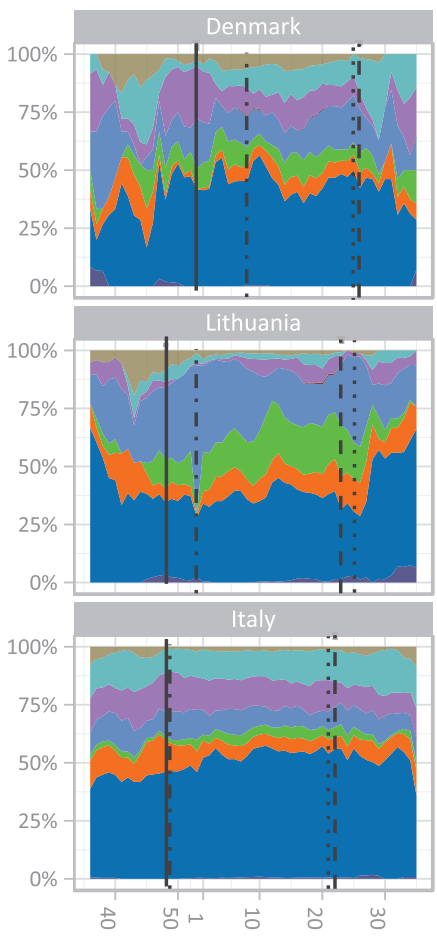

Week of year
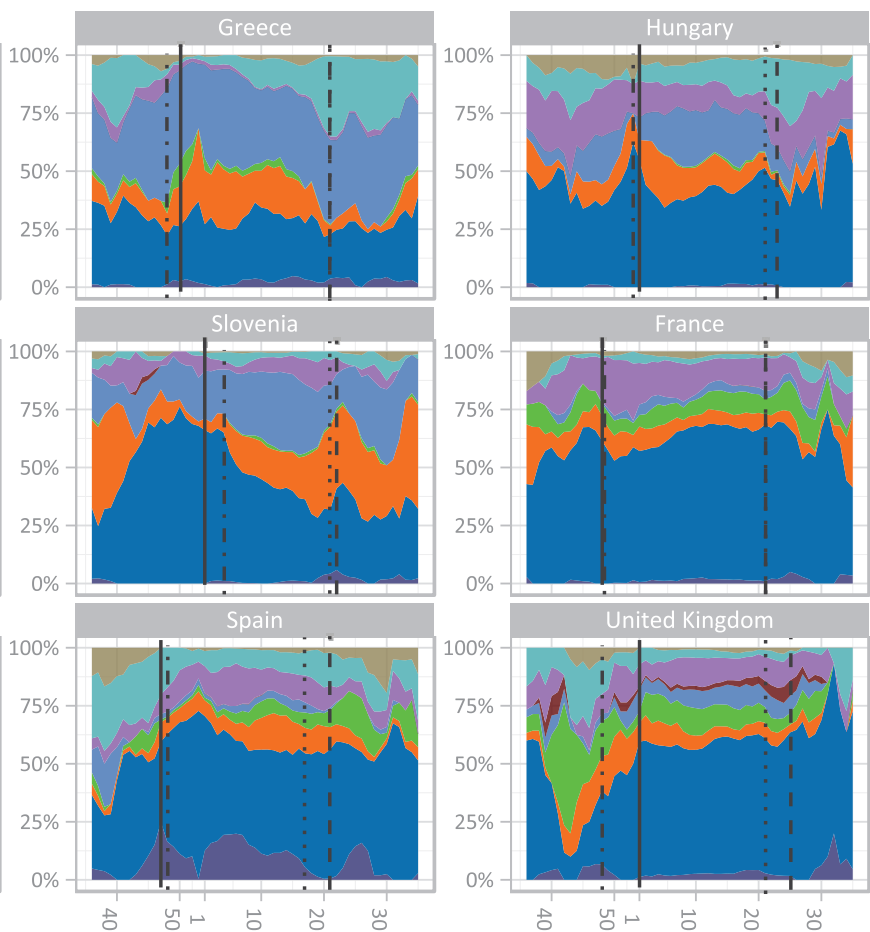

$100 \%$
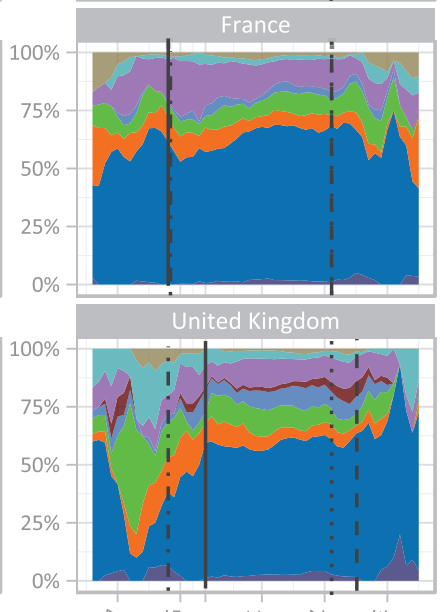

용

Case numbers were smoothed using a four-week moving average before conversion to proportions. The season legend shows vertical lines for the start and end of the rotavirus season, defined, respectively, by the median threshold method and through the questionnaire.

\section{Study area}

Twelve countries from EuroRotaNet were included in the study. Data from Austria, Belgium, Finland and Germany were excluded from the analysis because rotavirus vaccination was either routine or widespread $(335 \%)$ in these countries during the study period [13].

\section{Samples}

Study samples included rotavirus-positive faecal samples from mostly sporadic gastroenteritis cases; if associated with outbreaks, only a single sample per outbreak was submitted for routine diagnostic testing at sentinel participating EuroRotaNet laboratories and typed using standardised $\mathrm{G}$ and $\mathrm{P}$ typing methods $[12,14]$. Diagnostic testing protocols varied between countries $[12,14]$.

\section{Data and survey}

Details on case age, sex and country, specimen collection date and rotavirus genotyping results for a total of six rotavirus seasons spanning September 2007 to August 2013 were included in this study. Greece joined EuroRotaNet in 2008; therefore, for Greece only five rotavirus seasons were included in the analysis, spanning September 2008 to August 2013.
Data for each of the 12 countries were pooled for the study period. Age groups of cases (0-11 months, 12-23 months, 2-4 years and $\geq 5$ years) were constructed using date of birth and date of specimen collection. Genotypes were categorised as 'G1P[8]', 'genotypeconstellation 1 (Wa-like: G3P[8], G4P[8], G9P[8] and G12 P[8])', 'genotype-constellation 2 (DS-1-like: G2P[4] and G8P[4])', 'mixed and untypable', and less common genotypes were combined under the category 'other'. Although $\mathrm{G}_{1} \mathrm{P}[8]$ is considered part of genotype-constellation 1 (Wa-like), we grouped it separately because of its high prevalence across Europe [12]. A derived binary variable was constructed to denote weeks within a country's peak season and non-peak rotavirus seasons, and was developed by pooling country-specific weekly specimen frequencies over the study period to calculate the overall median weekly specimen frequency. We used the country-specific median value as a threshold for defining the start and end of the peak rotavirus season over the study period. Consequently, a weekly specimen frequency greater than or equal to the median identified weeks as in-season and a weekly frequency less than the median identified weeks as out-of-season. A consecutive period of two weeks 


\section{FIGURE 3}

Country-stratified crude and adjusted multinomial odds ratios for genotypes occurring out of season vs in season (model 2; $\mathrm{n}=39,786$ ) and for age group (model $4 ; \mathrm{n}=39,007)$, 12 European Union countries, September 2007-August 2013

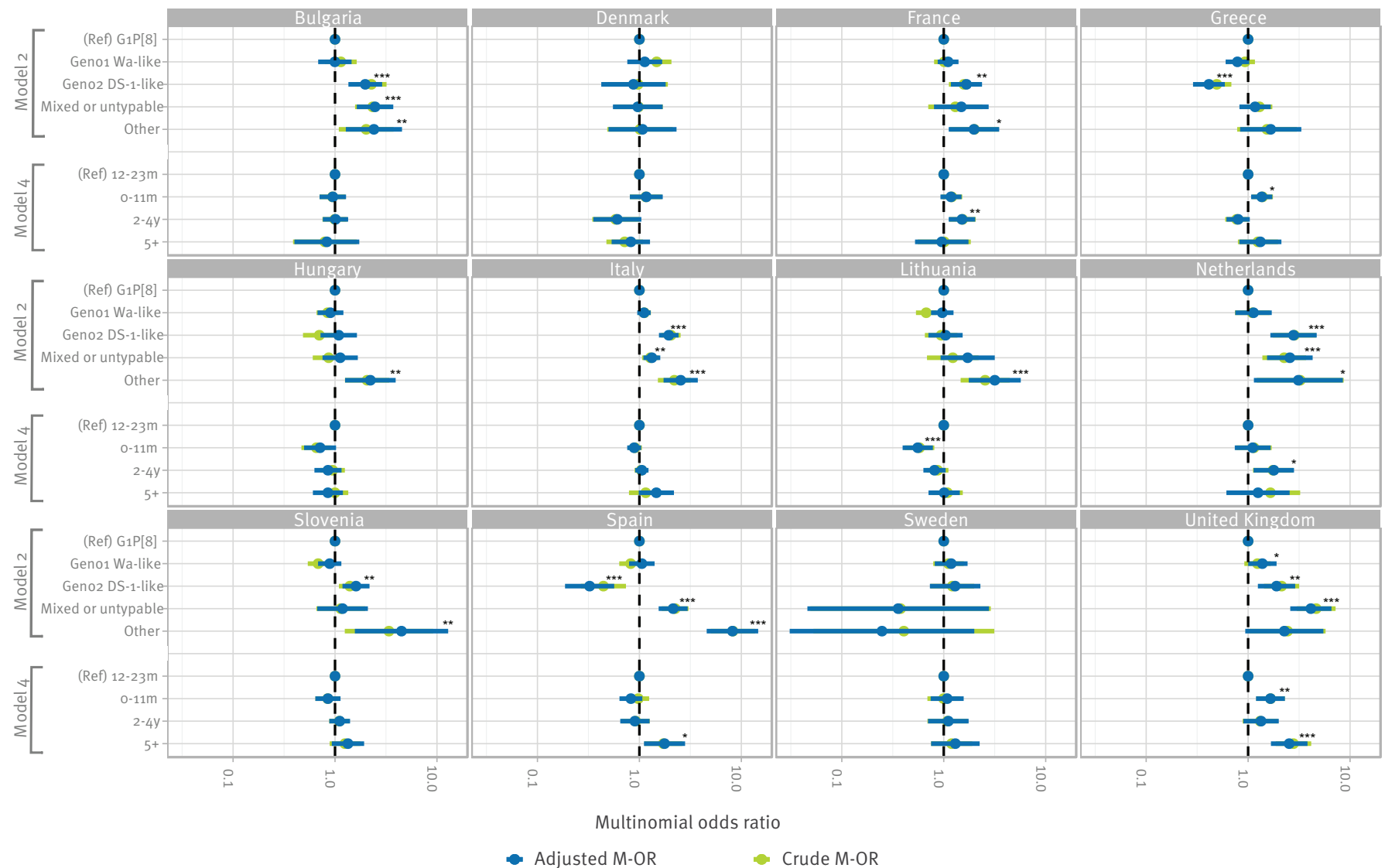

Multinomial odds ratios (M-OR) for out-of-season occurrence were estimated with multinomial logistic regression with the outcome variable genotype $\left(\mathrm{G}_{1} \mathrm{P}[8]\right.$ as the reference group) (model 2) or age group (12-23-month-olds as the reference group) (model 4$)$. Models were additionally adjusted for surveillance year (September-August) and either age group or genotype.

${ }^{*} p<0.05,{ }^{* \star} p<0.01, * \star * p<0.001$

Blue line: adjusted M-OR; green line: crude M-OR; Geno1 Wa-like: genotype-constellation 1 Wa-like; Geno2 DS-1-like: genotype-constellation 1 DS-1-like.

above or below the threshold was required to identify the beginning and end of a season to ensure season identification was robust to stochastic fluctuations.

To identify additional detail on country-specific inseason and out-of-season testing practices, we constructed a brief semi-structured questionnaire using SelectSurvey.Net [15]. The questionnaire was distributed to EuroRotaNet collaborators by email in July 2014 . The questionnaire included questions on duration of rotavirus season within the country, typical diagnostic testing practices, identification of changes to testing practices during the study period (including dates of any changes), positivity rate and proportion of positive samples typed. The questionnaire also asked for details on any age restrictions to testing or other algorithms that may have influenced testing and whether these may have changed between rotavirus seasons.

\section{Statistical analysis}

Models relating genotypes and age of cases to season To investigate differences in circulating genotypes and age of cases out of season vs in season, we fitted a series of mixed-effect multinomial logistic regression models with the two main outcomes: genotype group and age group of cases. Model fitting was based on variables identified a priori and used categorical variables for genotype group (reference group: G1P[8]), age group of the case (reference group: 12-23-month-olds), surveillance year (September to August) and country, and the binary season indicator was the covariate term of interest. The following adjusted models were then fit:

Genotype full model (model 1): genotype as the outcome variable; season, age group of case and surveillance year as covariates; and a random intercept for country. 


\section{FIGURE 4}

Rotavirus genotype diversity measured using Shannon's index and Simpson's index of diversity, with 95\% confidence intervals, by country, 12 European Union countries, pooled September 2007-August $2013(\mathrm{n}=39,786)$

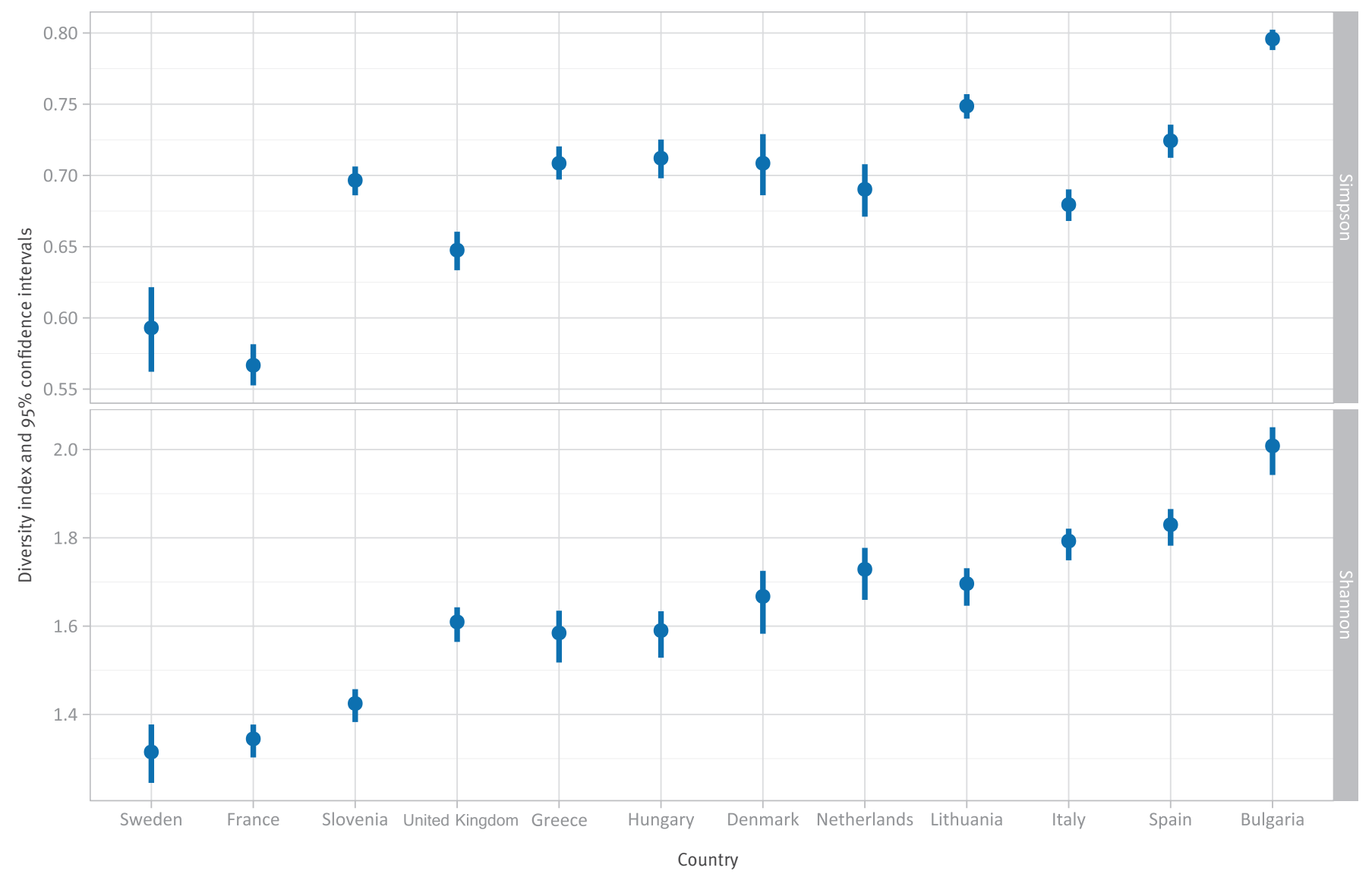

Genotype country-stratified model (model 2): model 1 but without a random intercept for country; effectively a series of country-specific multinomial logistic regressions.

Age group full model (model 3): age group of cases as the outcome variable; season, genotype and surveillance year as covariates; and a random intercept for country.

Age group country-stratified model (model 4): model 3 but without a random intercept for country; effectively a series of country-specific multinomial logistic regressions.

Each model was initially run as a univariate analysis including only the binary season indicator as the covariate term of interest. Multinomial odds ratios (M-OR; also referred to as RR ratios), 95\% confidence intervals $(\mathrm{Cl})$ and the associated $\mathrm{p}$ values for season were calculated from the Wald test. Results were considered significant at $p<0.05$. In supplementary analyses, mixed-effects multinomial logistic regression investigated the relationship between age group and genotype group regardless of season, therefore model 1 was re-run excluding season as a covariate (model 5).
Strain diversity

Rotavirus genotype diversity in the 12 European countries studied was compared using two established biodiversity indices, Simpson's index of diversity and Shannon's index [16]. Simpson's index of diversity (D) represents the probability that two randomly chosen rotavirus genotypes will have different $G$ and $P$ types and is calculated as $1-\lambda$, where $\lambda=\Sigma\left(p_{i}^{2}\right)$ and $p_{i}$ is the proportional abundance of a genotype $i$. Shannon's index $\left(H^{\prime}\right)$ quantifies the uncertainty in predicting the rotavirus genotype identity of an individual sample that is taken at random from the dataset and is calculated as $\mathrm{H}^{\prime}=-\Sigma\left(p_{i} \times \ln \left(p_{i}\right)\right)$. Confidence intervals were estimated using bootstrap resampling methodology and differences in season and out of season were compared for each country.

United Kingdom representativeness test

Linear regression was used to assess the representativeness of the seasonality of genotyped rotavirus data in comparison to all confirmed laboratory reports of rotavirus infection in the UK. The regression takes the form, $Y=a+\beta_{1} X_{1}+\beta_{2} X_{2}+\varepsilon$, where $Y$ is the number of confirmed laboratory reports of rotavirus infection, $X$ represents the covariates (number of genotyped rotavirus specimens and month of specimen), $a$ is the intercept term and $\varepsilon$ represents the error term. 
TABLE 1

Number of rotavirus specimens collected in season and out of season, by country, 12 European Union countries, September 2007-August $2013(\mathrm{n}=39,786)$

\begin{tabular}{|c|c|c|c|c|c|c|}
\hline \multirow[t]{2}{*}{ Country } & \multirow{2}{*}{$\begin{array}{c}\text { Total specimens } \\
\text { Number }\end{array}$} & \multicolumn{2}{|c|}{ In season } & \multicolumn{2}{|c|}{ Out of season } & \multirow{2}{*}{$\begin{array}{c}\text { In-season weeks } \\
\text { (calendar weeks) } \\
\text { Number }\end{array}$} \\
\hline & & Number & $\%$ & Number & $\%$ & \\
\hline Bulgaria & 2,627 & 2,296 & 87 & 331 & 13 & $31-17$ \\
\hline Denmark & 1,392 & 1,192 & 86 & 200 & 14 & $1-26$ \\
\hline France & 5,044 & 4,584 & 91 & 460 & 9 & $48-21$ \\
\hline Greece $^{a}$ & 2,115 & 1,447 & 68 & 668 & 32 & $50-21$ \\
\hline Hungary & 2,263 & 1,835 & 81 & 428 & 19 & $1-23$ \\
\hline Italy & 6,955 & 5,685 & 82 & 1,270 & 18 & $48-22$ \\
\hline Lithuania & 2,990 & 2,582 & 86 & 408 & 14 & $49-23$ \\
\hline The Netherlands & 2,508 & 2,346 & 94 & 162 & 6 & $48-22$ \\
\hline Slovenia & 2,779 & 2,272 & 82 & 507 & 18 & $1-22$ \\
\hline Spain & 4,609 & 4,227 & 92 & 382 & 8 & $47-21$ \\
\hline Sweden & 1,232 & 1,030 & 84 & 202 & 16 & $1-20$ \\
\hline United Kingdom & 5,272 & 5,014 & 95 & 258 & 5 & $1-25$ \\
\hline Total & 39,786 & 34,510 & 87 & 5,276 & 13 & NA \\
\hline
\end{tabular}

NA: not applicable.

a Data between September 2008 and August 2013.

All data handling and statistical analyses were performed using R Version 3.1.2. and Stata Version 14 $[17,18]$. The R packages 'Vegan' and 'boot' were used for analysis of genotype biodiversity [19,20]. Data tables are available through the EuroRotaNet website or available on request from the authors [10].

\section{Results}

\section{Descriptive statistics}

A total of 39,786 rotavirus-positive specimens from 12 countries were typed between September 2007 and August 2013. Rotavirus seasonality for genotyped rotavirus-positive specimens was variable across the countries studied (Figure 1). In the UK, the peak of the rotavirus season was well defined every year, typically occurring between weeks 10 and 12 . The representativeness test for the UK confirmed that the seasonal pattern of the typed rotavirus specimens was representative of laboratory-confirmed rotavirus cases in the UK (adjusted $R^{2}=0.75$ ). Table 1 shows the total number of typed specimens for each country, the number in season and out of season, and the number of weeks per year classified as in season. The proportion of specimens referred for typing that were collected in season ranged from $68 \%$ in Greece to $95 \%$ in the UK.

The predominant genotype overall was $\mathrm{G}_{1} \mathrm{P}[8]$, representing $48 \%$ of the specimens included in the analysis (range: $24 \%$ in Bulgaria to $63 \%$ in France). G1P[8] predominated in all countries except Greece and Bulgaria where the predominant genotypes were $\mathrm{G}_{4} \mathrm{P}[8]$ and $\mathrm{G}_{2} \mathrm{P}$ [4], respectively (Table 2). Children younger than five years contributed $93 \%$ of the specimens (range: $77 \%$ in Denmark to $97 \%$ in Bulgaria, France and Italy).
It is difficult to distinguish aberrant events due to the data's stochastic nature (Figure 1). However, some can been explained by outbreaks of particular genotypes. For instance in Spain, the increased incidence during the 2011/12 surveillance year was due to an outbreak of $\mathrm{G}_{12} \mathrm{P}[8]$ in the north-eastern province of Gipuzkoa.

\section{Genotypes in season and between rotavirus seasons}

Across all countries studied, when adjusting for country, surveillance year and age group, the adjusted multinomial odds ratio (aM-OR) of infection caused by strains with DS-1-like genotype-constellation $(\mathrm{aM}-\mathrm{OR}=1.25$; 95\% Cl: 1.13-1.37; $p<0.001)$, mixed or untypable genotypes (aM-OR $=1.55 ; 95 \% \mathrm{Cl}: 1.40-1.72 ; \mathrm{p}<0.001)$ and less common genotypes (group: 'other'; $\mathrm{aM}-\mathrm{OR}=2.11$; $95 \% \mathrm{Cl}: 1.78-2.51 ; \mathrm{p}<0.001)$ increased out of season relative to $G_{1} P[8]$, while infection caused by strains with Wa-like genotype constellation declined (aM$\mathrm{OR}=0.93 ; 95 \% \mathrm{Cl}: 0.86-1.00 ; \mathrm{p}=0.04)($ model 1$)$.

In country-stratified analyses (model 2), the proportional distribution of rotavirus genotypes varied by country (Figure 2). There were significant differences in the proportional representation of genotypes from specimens collected in season and out of season in 10 of the 12 countries studied. In these 10 counties, out-of-season specimens were more likely to belong to a less common genotype (group: 'other') than specimens collected in season (Figure 3). However, this was only significant in eight countries, with the highest $\mathrm{aM}-\mathrm{OR}$ observed in Spain $(\mathrm{aM}-\mathrm{OR}=8.18$; $95 \% \mathrm{Cl}: 4.57-14.64)$ and Slovenia (aM-OR = 4.49; $95 \% \mathrm{Cl}: 1.56-12.88)$. DS-1-like genotypes were significantly more likely to occur out of season in 


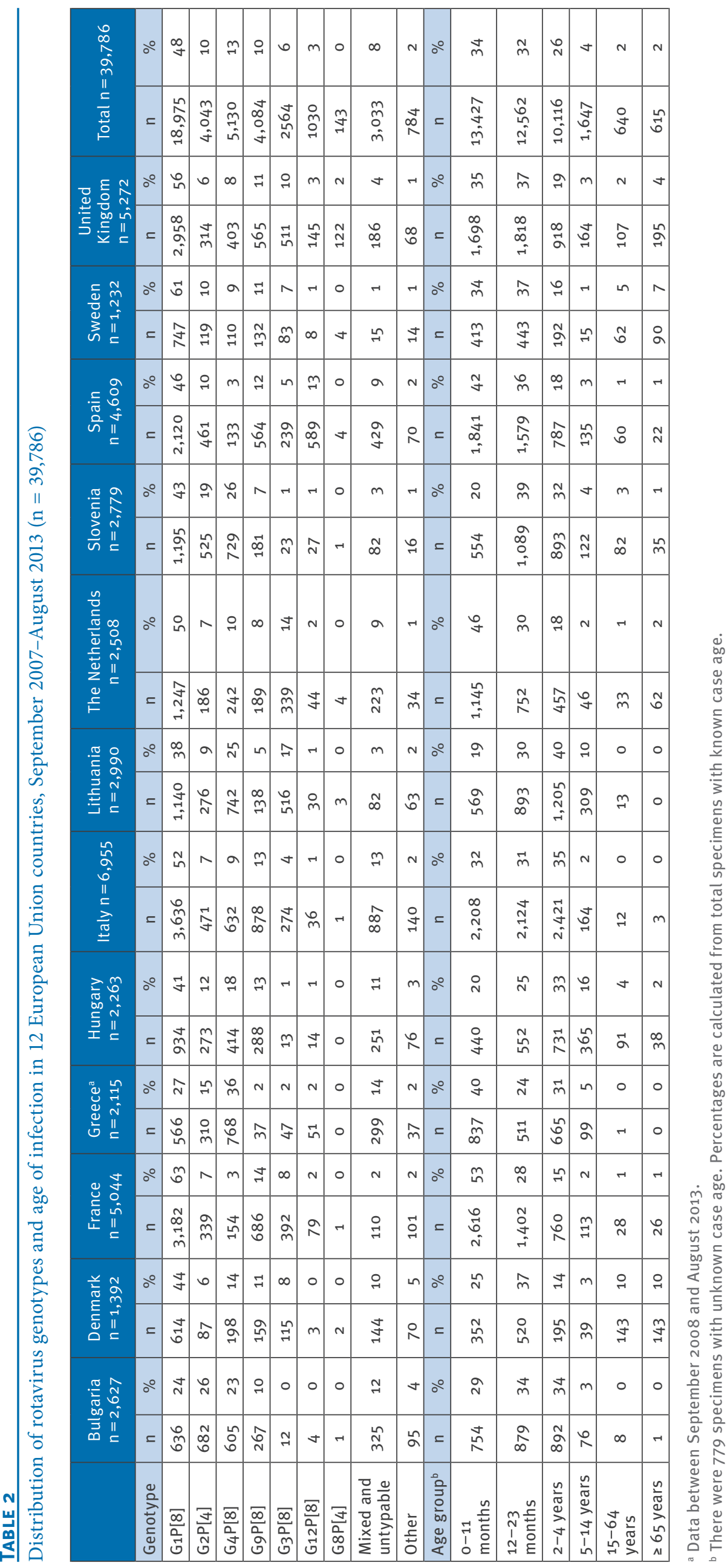


Bulgaria (aM-OR $=1.98 ; 95 \% \mathrm{Cl}: 1.35-2.90)$, France $(\mathrm{aM}-\mathrm{OR}=1.67 ; 95 \% \mathrm{Cl}: 1.18-2.37)$, Italy $(\mathrm{aM}-\mathrm{OR}=1.94$; 95\% Cl: $1.56-2.42$ ), the Netherlands (aM-OR $=2.79$; 95\% Cl: $1.65-4.71)$, Slovenia $(a M-O R=1.61 ; 95 \% \mathrm{Cl}$ : 1.18-2.18) and the UK (aM-OR $=1.90 ; 99 \mathrm{Cls:} 1.25-$ 2.90), whereas they were less likely to occur out of season in Spain (aM-OR $=0.32 ; 95 \% \mathrm{Cl}: 0.19-0.57)$ and Greece (aM-OR $=0.41 ; 95 \% \mathrm{Cl}: 0.29-0.59)$. Untypable and mixed genotypes had significantly higher proportional representation out of season in Bulgaria (aM$\mathrm{OR}=2.47 ; 95 \% \mathrm{Cl}: 1.63-3.73)$, Italy $(\mathrm{aM}-\mathrm{OR}=1.32$; 95\% Cl: $1.10-1.60$ ), the Netherlands (aM-OR $=2.57$; $95 \% \mathrm{Cl}: 1.53-4.29)$, Spain (aM-OR $=2.14$; $95 \% \mathrm{Cl}: 1.55-$ 2.98) and the UK (aM-OR $=4.13 ; 95 \% \mathrm{Cl}: 2.59-6.57)$. Only the UK (aM-OR $=1.38 ; 95 \% \mathrm{Cl}: 1.00-1.90)$ showed a significant change in the proportional representation of other genotype-constellation 1 (Wa-like) genotypes out of season compared with in season. Although Sweden and Denmark were the only two countries that did not show significant changes in genotype distribution out of season compared with in season, they had very different genotype distributions (Table 2 ).

\section{Age of cases in season and out of season}

Across all countries studied, when adjusting for country, surveillance year and genotype, the aM-OR of infection in two- to four-year-olds (aM-OR $=1.13 ; 95 \%$ $\mathrm{Cl}: 1.04-1.22 ; \mathrm{p}<0.01)$ and in those five years and older (aM-OR $=1.13 ; 95 \% \mathrm{Cl}: 1.00-1.27 ; \mathrm{p}=0.04)$ increased out of season relative to the younger children 12-23 months of age, while declining in those younger than 12 months (aM-OR $=0.92 ; 95 \% \mathrm{Cl}: 0.85-0.99 ; \mathrm{p}=0.03$ ) (model 3). Country-stratified analyses (model 4) showed that when adjusting for genotype and surveillance year, half of the countries experienced significant variation in the age group of cases out of season as compared with in season (Figure 3). Cases five years and older constituted a higher proportion of the out-ofseason than of the in-season cases in Greece, Italy, the Netherlands, Slovenia, Spain, Sweden and the UK. This difference was only significant in Spain $(\mathrm{aM}-\mathrm{OR}=1.76$; 95\% Cl: 1.11-2.81) and the UK (aM-OR $=2.53 ; 95 \% \mathrm{Cl}$ : 1.67-3.82). In France (aM-OR = 1.51; 95\% Cl: 1.12-2.04) and the Netherlands $(\mathrm{aM}-\mathrm{OR}=1.79 ; 95 \% \mathrm{Cl}: 1.13-$ 2.82), two- to four-year olds were significantly more commonly represented out of season compared with in season. Lithuania had significantly smaller proportions of cases $0-11$ months of age (aM-OR $=0.56 ; 95 \%$ $\mathrm{Cl}$ : 0.39-0.78) in season compared with out of season, whereas Greece (aM-OR $=1.36 ; 95 \% \mathrm{Cl}: 1.07-1.73)$ and the UK (aM-OR =1.66;95\% Cl: 1.20-2.30) had a significantly higher proportion of cases younger than 12 months out of season compared with in season.

\section{Relationship between age of cases and genotype group}

There was a significant association between increasing age and the genotypes causing disease regardless of season. Those five years and older were more likely to be infected with non-G1P[8] genotypes than those younger than five years (model 5 ). This was most pronounced in the DS-1-like genotype-constellation (aM-OR = 2.56; 95\% Cl: 2.27-2.90; $\mathrm{p}<0.001$ ), but also significant for mixed or untypable genotypes (aM$\mathrm{OR}=1.92 ; 95 \% \mathrm{Cl}: 1.65-2.23 ; \mathrm{p}<0.001)$, less common genotypes (group: 'other') (aM-OR 2.32; 95\% Cl: $1.79-3.02 ; p<0.001)$ and Wa-like genotype constellations $(\mathrm{aM}-\mathrm{OR}=1.15 ; 95 \% \mathrm{Cl}: 1.04-1.27 ; \mathrm{p}<0.01)$. The 0-11-months-old infants were also more likely than the reference group (12-23-month-olds) to be infected with mixed or untypable genotypes $(\mathrm{aM}-\mathrm{OR}=1.23$; $95 \%$ $\mathrm{Cl}$ : 1.11-1.35; $\mathrm{p}<0.001)$ and less common genotypes (group: 'other') $(\mathrm{aM}-\mathrm{OR}=1.30 ; 95 \% \mathrm{Cl}$ : 1.08-1.56; $p<0.01)$

\section{Genotype diversity}

Sweden and France had the lowest genotype diversity and Bulgaria the highest (Figure 4). Age group analysis showed that genotype diversity was highest in the age group five years and older in six of 12 countries based on Shannon's index and in eight of 12 countries based on Simpson's index of diversity. When cases five years and older were compared with the reference category of 12-23-month-olds, diversity was significantly higher in Shannon's index, Simpson's index of diversity or both indices in Denmark ( $\left.H^{\prime}: p=0.021 / D: p=0.585\right)$, Italy $\left(H^{\prime}\right.$ : $p<0.001 / D: p<0.001)$, the Netherlands (H': $p=0.192 / D$ : $p=0.003)$, Sweden (H': $p<0.001 / D: p<0.001)$ and the UK (H': $p<0.001 / D: p<0.001)$. When comparing genotype diversity in season with out-of-season genotype diversity, only Italy and the UK showed significant differences in genotype diversity. Both Shannon's index and Simpson's index of diversity showed significantly higher genotype diversity out of season in Italy ( $H^{\prime}$ : $p=0.012 / D: p<0.001)$ whereas only Simpson's index of diversity indicated significantly higher genotype diversity out of season in the UK $\left(H^{\prime}: p=0.098 / D: p=0.003\right)$.

\section{Survey}

All countries responded to the survey. Only Hungary indicated that they had reporting laboratories which did not test for rotavirus all year round. There was little variation in the temporal definition of the peak rotavirus season between the questionnaire responses and the statistical coding specified in the Methods chapter. The exceptions were Bulgaria and Denmark. The questionnaire response for Bulgaria specified no seasonality, whereas we identified weeks 31 to 17 for this analysis. For Denmark, the questionnaire response specified peak rotavirus season as March to June, while for the analysis, we defined it as weeks 1 to 26 (i.e. beginning in January).

Diagnostic tests used included enzyme-linked immunosorbent assay (ELISA), dual adenovirus/rotavirus rapid immunochromatographic tests (RIT), real-time RT-PCR, single rotavirus RIT, and electron microscopy. Dual RIT (9/12 responses) and ELISA (8/12 responses) were the most common tests. During the time period studied, it was reported that one laboratory in France had changed testing from latex agglutination to Dual RIT, 
and laboratories in four other countries had changed from ELISA to real-time RT-PCR or increased its use.

Age testing policies were variable across countries. Italy, Spain and the UK specified that they routinely test only children younger than five years, while other countries either included older children or tested all ages. Only one laboratory in France was identified as changing age group testing polices out of season. This laboratory specified that it changed from testing all ages to testing immunocompromised cases and children younger than five years only. In addition, a variety of factors were reported as influencing decision to test, but clinician request was selected in every survey response. Other common factors influencing decision to test included nosocomial outbreaks of acute gastroenteritis in a paediatric ward (10/12 responses) and diarrhoeal outbreaks in a nursery (8/12 responses). Apart from the aforementioned laboratory in France, respondents indicated that factors influencing testing for rotavirus were the same in season as out of season, and all countries stated that their decision to genotype did not vary in season and out of season.

\section{Discussion}

Significant differences in the circulating rotavirus genotypes in season compared with out of season were observed across the countries studied. Genotype $\mathrm{G}_{1} \mathrm{P}[8]$ was dominant in season but this dominance declined out of season in most countries, whereas the proportion of other less abundant genotypes increased out of season. Other than the dominance of $\mathrm{G}_{1} \mathrm{P}[8]$ in most countries, there was little consistency in genotype distribution across countries studied, highlighted by the country-to-country variation in genotype diversity and relative genotype dominance. For instance in Bulgaria, no genotype was identified as dominant, and the survey results further elucidated that Bulgaria does not appear to have a well-defined rotavirus season.

The analysis also showed that there were clear seasonal differences in the age distribution among rotavirus cases out of season vs in season. These differences were not consistent across all the countries studied. Generally, the proportion of cases five years and older increased out of season and in most countries, genotypes found in cases aged five years and older were more diverse than genotypes identified among younger age groups regardless of season. Relative to younger cases, cases aged at least five years were more likely to be infected with a non-G1P[8] genotype, in particular genotypes from the DS-1-like genotype constellation.

The relative decline of $\mathrm{G}_{1} \mathrm{P}[8]$ genotypes out of season is common in European countries and by definition coincides with a flattening of incidence, similar to countries with smoother incidence throughout the year, such as Bulgaria, where no single genotype is dominant. This pattern is also reflected in observations from countries which have introduced rotavirus vaccination, reinforcing the importance of understanding the pre-vaccine ecology of rotavirus infection across Europe for interpreting changes in rotavirus genotype distribution, seasonality and age of infection after vaccine introduction [21-24].

Seasonal and age group differences in the distribution of rotavirus genotypes may be driven by differential virus fitness among susceptible and partially immune hosts. Younger children, who are more susceptible, may be preferentially infected by the $\mathrm{G}_{1} \mathrm{P}[8]$ genotype, which given its predominance in most countries may be better adapted to the host or to transmission. The out-of-season decline in $\mathrm{G}_{1} \mathrm{P}[8]$ dominance may then be driven by the accumulation of homotypic immunity to $\mathrm{G}_{1} \mathrm{P}[8]$ in the community during the rotavirus season, reducing the number of susceptible hosts out of season and enabling the potentially less fit non-G1P[8] genotypes to infect those who have homotypic immunity from previous exposure to $\mathrm{G}_{1} \mathrm{P}[8]$ (24-60-month-olds may only have partial protection due to limited number of exposures) and older individuals infected with other genotypes to which cross-protection may be incomplete $[25,26]$. Indeed, a Mexican study showed that natural rotavirus infection reduces host susceptibility after each infection and that secondary infections are more likely to be caused by a different genotype than the one causing the first infection [25]. Furthermore, this explanation may be consistent with previous findings in which birth cohort effects were identified as potential drivers for differences in seasonality across the United States (US) [27].

Such differences between heterotypic and homotypic protection conferred by the dominant $\mathrm{G}_{1} \mathrm{P}[8]$ genotype support results from vaccine efficacy and observational studies of the monovalent Rotarix vaccine, which show that although the vaccine does protect against completely heterologous genotypes (e.g. G2P[4]), it may do so to a lesser extent [28-31].

The analysis also showed that mixed and untypable genotypes proportionally increased in a number of countries out of season. The types available for partially typed rotaviruses ( $G$ or $P$ type unobtainable) appear to be representative of the more commonly found types (typically $\mathrm{G}_{1}$ or $\mathrm{P}[8]$ ). Insufficient sensitivity of the typing procedures is the most likely cause for the typing failures [32]. These samples may, therefore, contain lower viral loads, which are likely to be associated with infections in previously exposed individuals with partial protection and/or subclinical infections.

Therefore, a plausible explanation for the increase in the proportional representation of older children and adults and of mixed and untypable genotypes out of season might be the accidental detection of an (asymptomatic) rotavirus infection in previously exposed individuals protected from severe RVGE, coinciding with infection by another pathogen causing gastrointestinal symptoms that has peak incidence in the summer months, such as some gastrointestinal bacterial 
pathogens. This could be supported by a study in the US that found that in adults admitted to hospital with diarrhoea, rotavirus was as commonly detected as bacterial gastrointestinal pathogens [33]. Furthermore, pre-vaccine studies suggest that there are high symptomatic and asymptomatic infection rates in adults regardless of epidemic season and that re-infection in adults persists across the year, which may suggest that older children and adults may be a reservoir from which the winter/spring paediatric epidemic emerges [34-36].

Our findings also suggest rapid genotype cycling from in-season to out-of-season periods and, as noted by Pitzer et al. [26], this could be caused by relatively stronger homotypic immunity than heterotypic immunity in the population, which renders the less common genotypes increased fitness, permitting them to persist in the population [26,37]. Moreover, age increases among RVGE cases as the predominant genotype declines, and the rapid cycling to less common genotypes out of season may explain the proportional increase in two- to four-year-olds and those five years and older seen in our analysis out of season $[26,27]$. However, an increase in those five years and older out of season may also be influenced by delayed transmission to this group because of mixing and contact patterns in younger children and infants. Additionally, the change to older age groups and less common genotypes out of season could potentially be related to importations associated with travel.

Interpretation of the proportional increase in specimens from those five years and older is, however, complicated by testing practices. The survey suggests that laboratories in some countries routinely test for rotavirus only in children younger than five years or, in some cases, those younger than 18 years, while limited testing occurs in older age groups. However, only one laboratory among the study countries reported changes in either age-specific testing procedures or clinician requests in season compared with out of season. Also, specifically in the UK, published guidance suggests a consistent testing algorithm all year, indicating that the reported variation in age of infection is representative [38].

Unfortunately, there is no apparent explanation for increases in the proportion of rotavirus-positive infants younger than 12 months out of season in Greece and the UK and for the decline in Lithuania. Findings are unlikely to be explained by seasonal birth rates as birth rate seasonality is similar in all the countries studied, suggesting that other factors, such as low heterotypic immunity conferred by previous infection, may be responsible [39].

We have described a number of potential hypotheses that may contribute to the observed differences in genotype and age distribution in season and out of season. However, we recognise this is not exhaustive and there may be other plausible hypotheses.

\section{Strengths and limitations}

Our analysis benefited from using an established surveillance system that has achieved consistency over a number of years. We supplemented our understanding of these data with a network-wide survey of testing practices. Nevertheless, there are limitations. Firstly, the sample size of rotavirus-positive samples typed was calculated based on detecting genotypes with a prevalence of at least $1 \%$ and, depending upon the country population size and estimated rates of rotavirus infection, are therefore not representative of the incidence of RVGE [11]. Secondly, it is unknown how many samples are referred for rotavirus diagnosis or how many are positive in routine diagnostic laboratories given that rotavirus is not a notifiable disease in many of the countries studied. For this reason we were unable to provide the proportion of positive samples each country submits for genotyping. Consequently we could not quantify the effect of sampling bias on out-of-season increases in less common genotypes, and the smaller number of cases out of season means that we must be aware of random variation when considering the findings. However, the study design helped to increase precision by pooling data over a number of seasons. Thirdly, data completeness of sex in the EuroRotaNet database was inconsistent across the countries studied. Previous analysis of EuroRotaNet data has shown no differences in genotype distribution between the sexes [11]. For these reasons sex was excluded from our models. Fourthly, the survey has shown that diagnostic procedures can vary slightly between countries and that a small number of laboratories have changed testing practices during the study period, which may have influenced the number of detected cases. However, a study in the UK found no association between number of laboratory reports and proportion of cases diagnosed by each diagnostic method [40]. Fifthly, even though countries included in the study had either low vaccination coverage $(<35 \%)$ or total absence of routine rotavirus vaccination [13], we have been unable to account for the effect of low-level vaccination in countries in which vaccine is available in regions and/or in the private health care sector, or the effect of routine vaccination in neighbouring countries on our findings. Finally, it is important to acknowledge that EuroRotaNet data are likely to be representative only of moderate to severe cases because in many countries, rotavirus is not a notifiable disease and because symptoms often resolve without healthcare contact.

\section{Conclusions}

This study shows that rotavirus genotype distribution in Europe is variable and that most countries included in this study experience variation in genotypes typed from specimens collected during the peak rotavirus season compared with the out-of-season periods. Changes in age of infection between peak rotavirus season and out-of-peak season may be due to lower 
cross-protection against heterotypic genotypes. These findings raise several questions about the genotype reservoirs and genotype persistence that may help direct future research to understand the temporal variability in the environment and in hosts. In addition, the true burden and epidemiology of rotavirus infections in adults and older children are not well understood due to age-exclusive testing policies, but the study further indicates that this could be critical to understanding re-infection and transmission that persists to re-ignite the epidemic season each year.

Finally, of the countries studied here, the UK has since introduced rotavirus vaccination into the childhood immunisation schedule. Critically, this work provides important pre-vaccine ecological data for the UK and other European countries introducing or expanding rotavirus vaccination programmes.

\section{Members of the EuroRotaNet}

Stephan Aberle (Department of Virology, Medical University of Vienna, Austria); Marc Van Ranst, Jelle Matthijnssens and Mark Zeller (Rega Institute for Medical Research, University of Leuven, Belgium); Zornitsa Mladenova (National Reference Laboratory of Enteroviruses, Department of Virology, NCIPD, Bulgaria); Sofie Midgley and Thea Kølsen Fischer (Virology Surveillance and Research unit, Statens Serum Institut, Copenhagen, Denmark); Vassiliki P Syriopoulou and Dimitra M Koukou (First Department of Pediatrics, Athens University, Aghia Sophia Children's Hospital, Athens, 11527, Greece); Krisztián Bányai and Renáta Dóró (Institute for Veterinary Medical Research, Centre for Agricultural Research, Hungarian Academy of Sciences, Budapest, Hungary); Jérôme Kaplon and Pierre Pothier (National Reference Centre for enteric viruses - Laboratory of virology, University Hospital of Dijon, Dijon, France); Leena Maunula (Department. of Food Hygiene and Environmental Health, University of Helsinki, Helsinki, Finland); Franco Maria Ruggeri and Lucia Fiore (Istituto Superiore di Sanità, Rome, Italy), Vytautas Usonis and Inga Ivaskeviciene (Vilnius University, Faculty of Medicine, Clinic of Children Diseases, Vilnius, Lithuania), Harry Vennema and Annelies Kroneman (National Institute for Public Health and the Environment, Bilthoven, The Netherlands); Mateja Poljsak-Prijatelj and Andrej Steyer (University of Ljubljana, Faculty of Medicine, Institute of Microbiology and Immunology, Ljubljana, Slovenia); Javier Buesa (University of Valencia, Valencia, Spain), Gustavo Cilla and Milagroasa Montes (Servicio de Microbiología Clínica, Hospital Universitario Donostia, San Sebastián, Spain (2010-2011); Mia Brytting and Lottie Schloss (Public Health Agency of Sweden, Solna, Sweden); Andreas Mas Marques (RobertKoch-Institut, Berlin, Germany); David James Allen and Sameena Nawaz (Virus Reference Department, Public Health England, UK)

\section{Acknowledgements}

We are indebted to all physicians and microbiologists that help to collect clinical and microbiological data for rotavirus patients.

Funding: Funding for this study was provided by GlaxoSmithKline (GSK) Biologicals SA and Sanofi PasteurMSD (SPMSD). GlaxoSmithKline Biologicals SA and SPMSD were provided the opportunity to review a version of this manuscript for factual accuracy but the authors are solely responsible for final content and interpretation. The authors received no financial support or other form of compensation related to the development of the manuscript. JMR was supported by The Farr Institute for Health Informatics Research (MRC grant: MR/M0501633/1). VEP was supported by the Bill and Melinda Gates Foundation. MIG receives support from The Wellcome Trust and the National Institute for Health Research Health Protection Research Unit in Gastrointestinal Infections at the University of Liverpool. DH was supported by the University of Liverpool.

\section{Conflict of interest}

Rotarix is developed and licensed by GSK Biologicals. NC has recieved research grant support from GSK Biologicals and honoraria for participation in GSK Rotavirus Vaccine Advisory Board Meetings.

\section{Authors' contributions}

DH participated in study design, developed the survey, performed data management, conducted the analysis and wrote the manuscript. EuroRotaNet members contributed to study design and data collection. RV contributed to study design and survey design. JMR contributed to the analysis. VEP contributed to the analysis. NF participated in study design and contributed to the analysis. NC contributed to interpretation of data. MIG conceived of the study; contributed to survey design and data collection. All authors contributed to the interpretation of the data, drafting the article, and final approval of the version to be published. No person or persons other than the authors listed have contributed significantly to the study or manuscript preparation.

\section{References}

1. Tate JE, Burton AH, Boschi-Pinto C, Steele AD, Duque J, Parashar UD, et al. 2008 estimate of worldwide rotavirusassociated mortality in children younger than 5 years before the introduction of universal rotavirus vaccination programmes: a systematic review and meta-analysis. Lancet Infect Dis. 2012;12(2):136-41. DOI: 10.1016/S14733099(11)70253-5 PMID: 22030330

2. Tam CC, Rodrigues LC, Viviani L, Dodds JP, Evans MR, Hunter $P R$, et al. Longitudinal study of infectious intestinal disease in the UK (IID2 study): incidence in the community and presenting to general practice. Gut. 2012;61(1):69-77. DOI: $10.1136 /$ gut.2011.238386 PMID: 21708822

3. Harris JP, Jit M, Cooper D, Edmunds WJ. Evaluating rotavirus vaccination in England and Wales. Part I. Estimating the burden of disease.Vaccine. 2007;25(20):3962-70. DOI: 10.1016/j. vaccine.2007.02.072 PMID: 17395343

4. Matthijnssens J, Otto PH, Ciarlet M, Desselberger U, Van Ranst M, Johne R. VP6-sequence-based cutoff values as a criterion for rotavirus species demarcation. Arch Virol. 2012;157(6):117782. DOI: 10.1007/s00705-012-1273-3 PMID: 22430951

5. Desselberger U. Rotaviruses: basic facts. In: Gray J, Desselberger U, editors. Rotaviruses methods and protocols. New Jersey: Humana Press; 2000. p. 1-8.

6. Matthijnssens J, Ciarlet M, Rahman M, Attoui H, Bányai K, Estes MK, et al. Recommendations for the classification of group A rotaviruses using all 11 genomic RNA segments. Arch Virol. 2008;153(8):1621-9. DOI: 10.1007/s00705-008-0155-1 PMID: 18604469

7. European Centre for Disease Prevention and Control (ECDC). Vaccine schedule: Recommended immunisations for rotavirus infection. Stockholm: ECDC. [Accessed: 2 Mar 2015]. Available from: http://vaccine-schedule.ecdc.europa.eu/Pages/ Scheduler.aspx

8. Matthijnssens J, Nakagomi O, Kirkwood CD, Ciarlet M, Desselberger U, Van Ranst M. Group A rotavirus universal mass vaccination: how and to what extent will selective pressure influence prevalence of rotavirus genotypes?Expert Rev Vaccines. 2012;11(11):1347-54. DOI: 10.1586/erv.12.105 PMID: 23249234

9. Zeller M, Rahman M, Heylen E, De Coster S, De Vos S, Arijs I, et al. Rotavirus incidence and genotype distribution before and after national rotavirus vaccine introduction 
in Belgium. Vaccine. 2010;28(47):7507-13. DOI: 10.1016/j. vaccine.2010.09.004 PMID: 20851085

10. EuroRotaNet. [Accessed: 11 Nov 2014]. Available from: http:// www.eurorota.net/

11. Iturriza-Gómara M, Dallman T, Bányai K, Böttiger B, Buesa J, Diedrich S, et al. Rotavirus surveillance in Europe, 2005-2008: web-enabled reporting and real-time analysis of genotyping and epidemiological data. J Infect Dis. 2009;200(s1) Suppl 1;S215-21. DOI: 10.1086/605049 PMID: 19821712

12. Iturriza-Gómara M, Dallman T, Bányai K, Böttiger B, Buesa J, Diedrich S, et al. Rotavirus genotypes co-circulating in Europe between 2006 and 2009 as determined by EuroRotaNet, a panEuropean collaborative strain surveillance network. Epidemiol Infect. 2011;139(6):895-909. DOI: 10.1017/So950268810001810 PMID: 20707941

13. Parez N, Giaquinto C, Du Roure C, Martinon-Torres F, Spoulou $V$, Van Damme $P$, et al. Rotavirus vaccination in Europe: drivers and barriers. Lancet Infect Dis. 2014;14(5):416-25. DOI: 10.1016/S1473-3099(14)70035-0 PMID: 24758998

14. EuroRotaNet. Rotavirus detection and typing methods. UK: EuroRotaNet. [Accessed: 25 Nov 2014] p. 10-25. Available from: http://www.eurorota.net/docs.php

15. SelectSurvey.Net. Overland Park: Classapps; 2012. Available from: www.classapps.com/SelectSurveyNETOverview.asp

16. Legendre P, Legendre LFJ. Numerical ecology, Volume 24. 3 edition. Amsterdam: Elsevier; 2012. 1006 p.

17. R Core Team. R: a language and environment for statistical computing. Vienna: R Foundation for Statistical Computing; 2013. Available from: http://www.R-project.org

18. StataCorp. Stata statistical software: Release 14. College Station: StataCorp LP; 2015. Available from: http://www.Stata. com/

19. Oksanen J, Blanchet FG, Kindt R, Legendre P, Minchin PR, O'Hara RB, et al. vegan: community ecology package. 2013 [Accessed: 10 Nov 2014]. Available from: http://cran.r-project. org/web/packages/vegan/index.html

20. Gardener M. Community ecology : analytical methods using R and Excel. Exeter: Pelagic Publishing; 2014.

21. Braeckman T, Van Herck K, Raes M, Vergison A, Sabbe M, Van Damme P. Rotavirus vaccines in Belgium: policy and impact. Pediatr Infect Dis J. 2011;30(1) Suppl;S21-4. DOI: 10.1097/ INF.ob013e3181fefc51 PMID: 21183836

22. Cortese MM, Tate JE, Simonsen L, Edelman L, Parashar UD. Reduction in gastroenteritis in United States children and correlation with early rotavirus vaccine uptake from national medical claims databases.Pediatr Infect Dis J. 2010;29(6):48994.PMID: 20354464

23. Dóró R, László B, Martella V, Leshem E, Gentsch J, Parashar U, et al. Review of global rotavirus strain prevalence data from six years post vaccine licensure surveillance: is there evidence of strain selection from vaccine pressure? Infect Genet Evol. 2014;28:446-61. DOI: 10.1016/j.meegid.2014.08.017 PMID: 25224179

24. Gómez MM, Carvalho-Costa FA, Volotão E de M, Rose TL, da Silva MFM, Fialho AM, et al. Prevalence and genomic characterization of $\mathrm{G}_{2} \mathrm{P}$ [4] group A rotavirus strains during monovalent vaccine introduction in Brazil. Infect Genet Evol. 2014;28:486-94. DOI: 10.1016/j.meegid.2014.09.012 PMID: 25239525

25. Velázquez FR, Matson DO, Calva JJ, Guerrero L, Morrow AL, Carter-Campbell $S$, et al. Rotavirus infections in infants as protection against subsequent infections. N Engl J Med. 1996;335(14):1022-8. DOI: 10.1056/NEJM199610033351404 PMID: 8793926

26. Pitzer VE, Patel MM, Lopman BA, Viboud C, Parashar UD, Grenfell BT. Modeling rotavirus strain dynamics in developed countries to understand the potential impact of vaccination on genotype distributions.Proc Natl Acad Sci USA 2011;108(48):19353-8. DOI: 10.1073/pnas.1110507108 PMID: 22084114

27. Pitzer VE, Viboud C, Simonsen L, Steiner C, Panozzo CA, Alonso WJ, et al. Demographic variability, vaccination, and the spatiotemporal dynamics of rotavirus epidemics. Science. 2009;325(5938):290-4. DOI: 10.1126/science.1172330 PMID: 19608910

28. Ruiz-Palacios GM, Pérez-Schael I, Velázquez FR, Abate $H$, Breuer T, Clemens SC, et al. Safety and efficacy of an attenuated vaccine against severe rotavirus gastroenteritis. N Engl J Med. 2006;354(1):11-22. DOI: 10.1056/NEJMoa052434 PMID: 16394298

29. Velasquez DE, Parashar UD, Jiang B. Strain diversity plays no major role in the varying efficacy of rotavirus vaccines: an overview.Infect Genet Evol. 2014;28:561-71. DOI: 10.1016/j. meegid.2014.10.008 PMID: 25460825
30. Braeckman T, Van Herck K, Meyer N, Pirçon J-Y, SorianoGabarró M, Heylen E, et al. Effectiveness of rotavirus vaccination in prevention of hospital admissions for rotavirus gastroenteritis among young children in Belgium: case-control Study. BMJ. 2012;345(augo8 1):e4752. DOI: 10.1136/bmj.e4752 PMID: 22875947

31. Matthijnssens J, Zeller M, Heylen E, De Coster S, Vercauteren J, Braeckman T, et al. Higher proportion of $\mathrm{G}_{2} \mathrm{P}[4]$ rotaviruses in vaccinated hospitalized cases compared with unvaccinated hospitalized cases, despite high vaccine effectiveness against heterotypic $\mathrm{G}_{2} \mathrm{P}[4]$ rotaviruses. Clin Microbiol Infect. 2014;20(10):0702-10. DOI: 10.1111/1469-0691.12612 PMID: 24580887

32. Aladin F, Nawaz S, Iturriza-Gómara M, Gray J. Identification of $\mathrm{G} 8$ rotavirus strains determined as $\mathrm{G} 12$ by rotavirus genotyping PCR: updating the current genotyping methods.] Clin Virol. 2010;47(4):340-4. DOI: 10.1016/j.jcV.2010.01.004 PMID: 20138804

33. Anderson EJ, Katz BZ, Polin JA, Reddy S, Weinrobe MH, Noskin GA. Rotavirus in adults requiring hospitalization.J Infect. 2012;64(1):89-95. DOI: 10.1016/j.jinf.2011.09.003 PMID: 21939687

34. Cox MJ, Medley GF. Serological survey of anti-group A rotavirus IgM in UK adults. Epidemiol Infect. 2003;131(1):719-26. DOI: 10.1017/So950268803008720 PMID: 12948372

35. Nakajima H, Nakagomi T, Kamisawa T, Sakaki N, Muramoto K, Mikami T, et al. Winter seasonality and rotavirus diarrhoea in adults. Lancet. 2001;357(9272):1950. DOI: 10.1016/S01406736(00)05086-8 PMID: 11425422

36. Phillips G, Lopman B, Rodrigues LC, Tam CC. Asymptomatic rotavirus infections in England: prevalence, characteristics, and risk factors.Am J Epidemiol. 2010;171(9):1023-30. DOI: 10.1093/aje/kwq050 PMID: 20392863

37. Parra GI. Seasonal shifts of group A rotavirus strains as a possible mechanism of persistence in the human population.J Med Virol. 2009;81(3):568-71. DOI: 10.1002/jmv.21423 PMID: 19152398

38. Public Health England. UK standards for microbiology investigations $\mathrm{S}$ 7: gastroenteritis and diarrhoea. London: Public Health England; 2013. p. 1-20. Available from: https://www.gov.uk/government/publications/ smi-s-7-gastroenteritis-and-diarrhoea

39. UNdata. Live births by month of birth. New York: United Nations Statistics Division. [Accessed: 26 Mar 2015]. Available from: http://data.un.org/Data. asp $\mathrm{x}$ d=POP\& $\mathrm{f}=$ tableCode $\% 3 \mathrm{~A} 55$

40. Atchison CJ, Lopman BA, Harris CJ, Tam CC, Iturriza Gómara $M$, Gray JJ. Clinical laboratory practices for the detection of rotavirus in England and Wales: can surveillance based on routine laboratory testing data be used to evaluate the impact of vaccination?Euro Surveill. 2009;14(20):pii= 19217.PMID: 19460285

\section{License and copyright}

This is an open-access article distributed under the terms of the Creative Commons Attribution (CC BY 4.0) Licence. You may share and adapt the material, but must give appropriate credit to the source, provide a link to the licence, and indicate if changes were made.

This article is copyright of the authors, 2016. 